\title{
Effect of Treat-to-target Strategies Aiming at Remission of Arterial Stiffness in Early Rheumatoid Arthritis: A Randomized Controlled Study
}

\author{
Lydia Ho-Pui Tam, Qing Shang, Edmund Kwok-Ming Li, Priscilla Ching-Han Wong, \\ Kitty Yan Kwok, Emily Wai-Lin Kun, Isaac Cheuk-Wan Yim, Violet Ka-Lai Lee, \\ Ronald Man-Lung Yip, Steve Hin-Ting Pang, Virginia Weng-Nga Lao, Queenie Wah-Yan Mak, \\ Isaac Tsz-Ho Cheng, Xerox Sze-Lok Lau, Tena Ka-Yan Li, Tracy Yaner Zhu, Alex Pui-Wai Lee, \\ and Lai-Shan Tam
}

\begin{abstract}
Objective. To determine the efficacy of 2 tight control treatment strategies aiming at Simplified Disease Activity Score (SDAI) remission (SDAI $\leq 3.3$ ) compared to 28-joint count Disease Activity Score (DAS28) remission (DAS28 < 2.6) in the prevention of arterial stiffness in patients with early rheumatoid arthritis (RA).

Methods. This was an open-label study in which 120 patients with early RA were randomized to receive 1 year of tight control treatment. Group $1(n=60)$ aimed to achieve SDAI $\leq 3.3$ and Group 2 $(\mathrm{n}=60)$, DAS2 $8<2.6$. Pulse wave velocity (PWV) and augmentation index (AIx) were measured at baseline and 12 months. A posthoc analysis was also performed to ascertain whether achieving sustained remission could prevent progression in arterial stiffness.

Results. The proportions of patients receiving methotrexate monotherapy were significantly lower in Group 1 throughout the study period. At 12 months, the proportions of patients achieving DAS28 and SDAI remission, and the change in PWV and AIx, were comparable between the 2 groups. In view of the lack of differences between the 2 groups, a posthoc analysis was performed at Month 12, including all 110 patients with PWV, to elucidate the independent predictors associated with the change in PWV. Multivariate analysis revealed that achieving sustained DAS28 remission at months 6, 9, and 12 and a shorter disease duration were independent explanatory variables associated with less progression of PWV.

Conclusion. With limited access to biologic disease-modifying antirheumatic drugs, treatment efforts toward DAS28 and SDAI remission had similar effects in preventing the progression of arterial stiffness at 1 year. However, achieving sustained DAS28 remission was associated with a significantly greater improvement in PWV. [Clinical Trial registration: Clinicaltrial.gov NCT01768923.] (First Release May 15 2018; J Rheumatol 2018;45:1229-39; doi:10.3899/jrheum.171128)
\end{abstract}

Key Indexing Terms:

EARLY RHEUMATOID ARTHRITIS ARTERIAL STIFFNESS PULSE WAVE VELOCITY AUGMENTATION INDEX

REMISSION

From the Department of Medicine and Therapeutics, and Department of Orthopaedics and Traumatology, The Chinese University of Hong Kong; Department of Medicine, Queen Elizabeth Hospital; Department of Medicine, Tai Po Hospital; Department of Medicine, Tseung Kwan $O$ Hospital; Department of Medicine, Pamela Youde Nethersole Eastern Hospital; Department of Medicine, Kwong Wah Hospital, Hong Kong Funding support came from the Health and Medical Research Fund (HMRF Project No. 10110071).

L.H. Tam, MBChB, Department of Medicine and Therapeutics, The Chinese University of Hong Kong; Q. Shang, Research Assistant Professor, PhD, Department of Medicine and Therapeutics, The Chinese University of Hong Kong; E.K. Li, Professor, MD, Department of Medicine and Therapeutics, The Chinese University of Hong Kong; P.C. Wong, Associate Consultant, FHKAM, Department of Medicine and

Therapeutics, The Chinese University of Hong Kong; K.Y. Kwok, Associate Consultant, FHKAM, Department of Medicine, Queen Elizabeth Hospital; E.W. Kun, Consultant, FRCP, Department of Medicine, Tai Po Hospital; I.C. Yim, Associate Consultant, FRCP, Department of Medicine, Tseung Kwan O Hospital; V.K. Lee, Associate Consultant, FHKAM, Department of Medicine, Pamela Youde Nethersole Eastern Hospital; R.M. Yip,
Associate Consultant, FRCP, Department of Medicine, Kwong Wah Hospital; S.H. Pang, Associate Consultant, FHKAM, Department of Medicine, Kwong Wah Hospital; V.W. Lao, Associate Consultant, FHKAM, Department of Medicine, Kwong Wah Hospital; Q.W. Mak, Research Assistant, PgD, Department of Medicine and Therapeutics, The Chinese University of Hong Kong; I.T. Cheng, Research Assistant, MSc, Department of Medicine and Therapeutics, The Chinese University of Hong Kong; X.S. Lau, Research Assistant, MPH, Department of Medicine and Therapeutics, The Chinese University of Hong Kong; T.K. Li, Nursing Officer, BN, Department of Medicine and Therapeutics, The Chinese University of Hong Kong; T.Y. Zhu, scientific officer, PhD, Department of Orthopaedics and Traumatology, The Chinese University of Hong Kong; A.P. Lee, Assistant Professor, MD, Department of Medicine and Therapeutics, The Chinese University of Hong Kong; L.S. Tam, Professor, $M D$, Department of Medicine and Therapeutics, The Chinese University of Hong Kong.

Address correspondence to Prof. L.S. Tam, Department of Medicine and Therapeutics, The Prince of Wales Hospital, The Chinese University of Hong Kong, Shatin, Hong Kong.E-mail: lstam@cuhk.edu.hk Accepted for publication February 12, 2018.

Personal non-commercial use only. The Journal of Rheumatology Copyright (C) 2018. All rights reserved. 
Rheumatoid arthritis (RA) is associated with a substantially increased risk of cardiovascular disease (CVD), leading to a 1.5-fold increased standardized mortality rate compared with the general population ${ }^{1}$. Studies have demonstrated the association of a higher inflammatory burden with increased CVD risk in $\mathrm{RA}^{2,3}$, suggesting that the increased levels of proinflammatory cytokines can elicit a systemic inflammatory state that accelerates atherosclerosis conducive to increased CV risk.

Rapid attainment of remission by tight control protocolized treatment strategy can halt joint damage and reverse disability irrespective of the type of disease-modifying antirheumatic drugs (DMARD), combination conventional synthetic (cs-) or biological (b-) DMARD ${ }^{4}$. The most frequently used definitions of remission have involved a 28 -joint count Disease Activity Score (DAS28) of $<2.6$. This definition better represents minimal disease activity than remission ${ }^{5}$. Based on the ability to predict nonprogression of articular disease regarding patient function and radiographic progression, the 2011 American College of Rheumatology (ACR)/European League Against Rheumatism (EULAR) criteria for remission were more stringent, to be used in clinical trials in $\mathrm{RA}^{6}$. There is a need for interventional trials with $\mathrm{CV}$ endpoints to investigate whether achievement of remission according to the new ACR/EULAR definition could reduce $\mathrm{CV}$ risk in RA. Further, it remains to be tested whether the new definition of remission, which reflects a more rigorous control of disease inflammation than the DAS28-based definition, could in turn translate into a more favorable CV outcome.

Increased number and duration of flares over time and cumulative RA disease activity contribute to the risk of $\mathrm{CVD}^{7}$. Effective immunosuppression using various bDMARD can improve arterial stiffness as reflected by a decrease in pulse wave velocity (PWV) or augmentation index $(\mathrm{AIx})^{8,9}$. From these standpoints, one can argue that a more rigorous and sustained control of inflammation might be associated with a more favorable $\mathrm{CV}$ outcome in patients with RA. Whether achievement of sustained remission could reduce CVD risk in RA has, to our knowledge, never been studied.

We hypothesized that tight control treatment aiming at the 2011 ACR/EULAR definition of remission reduces arterial stiffness to a greater extent than treatment aiming at minimal disease activity (DAS28 < 2.6) in patients with early RA. The aim of our study was to investigate the effect of these 2 treatment strategies on arterial stiffness in early RA. A posthoc analysis was also performed to ascertain whether achieving sustained remission can prevent progression in arterial stiffness in patients with early RA.

\section{MATERIALS AND METHODS}

Subjects. This was a 1-year, hospital-based, open-label, randomized controlled trial (RCT; Clinicaltrial.gov NCT01768923). There were 120 patients recruited from the rheumatology clinic of the Prince of Wales
Hospital (PWH), Queen Elizabeth Hospital (QEH), and 5 other regional hospitals from December 2012 to December 2015. The study protocol was in compliance with the Declaration of Helsinki and the International Conference on Harmonisation Good Clinical Practice. The study was approved by the Kowloon Central Cluster Research Ethics Committee for QEH (KC-KE13-0040/FR-1), Kowloon East Cluster Research Ethics Committee for Tseung Kwan O Hospital (KC-KE13-0040/FR-1), Kowloon West Cluster Research Ethics Committee for Kwong Wah Hospital [(KW/EX-14-052(73-07)], and The Joint Chinese University of Hong Kong-New Territories East Cluster Clinical Research Ethics Committee for PWH and Tai Po Hospital (CRE 2011.483-T). All patients provided written consent.

Inclusion and exclusion criteria. Patients were eligible if they fulfilled the following inclusion criteria: (1) 2010 ACR/EULAR classification criteria for $\mathrm{RA}^{10}$; (2) symptoms onset $<2$ years; (3) active disease (DAS28 $\geq 3.2$ ); and (4) positivity for rheumatoid factor or anticyclic citrullinated peptide antibodies. Patients were excluded if they had the following: (1) history of overt CVD; (2) aspirin, HMG-CoA reductase inhibitors (statins), or angiotensin-converting enzyme (ACE) inhibitors; (3) severe renal impairment (glomerular filtration rate $<30 \mathrm{ml} / \mathrm{min} / 1.73 \mathrm{~m}^{2}$ ); (4) previous treatment with bDMARD; or (5) prednisolone at a dose of $>10 \mathrm{mg} /$ day.

Intervention. All participants received 1-year tight control treatment at the PWH and QEH with the aim of achieving treatment target (Supplementary Figures 1 and 2, available with the online version of this article). Participants were randomly assigned to 2 arms. Group 1 aimed at the 2011 ACR/EULAR definition of remission (SDAI $\leq 3.3)^{6}$. Group 2 aimed at minimal disease activity using C-reactive protein (CRP; DAS28-CRP < 2.6). Patients in Group 1 were started with csDMARD or bDMARD if they had moderate to high disease activity and poor prognostic factors (Supplementary Figure 1 , available with the online version of this article). Patients in Group 2 were started with methotrexate (MTX) monotherapy, and followed with csDMARD or bDMARD if they failed to achieve target (Supplementary Figure 2). Assessment of the efficacy was performed every 3 months. If a patient could not achieve treatment goal within 3 months of therapy, the attending rheumatologist would adjust therapy by proceeding to the next step according to the protocol, unless the patient declined or toxic effects precluded this approach. Statins and ACE inhibitors were prohibited during the whole study because they might have altered the vascular studies results.

Clinical assessments. At baseline and then every 3 months up to 1 year, the number of tender and swollen joints (0-28), numeric rating scale for pain, patient's and physician's global assessments, Health Assessment Questionnaire (HAQ), erythrocyte sedimentation rate $\left(\mathrm{mm} / \mathrm{h}^{1 \mathrm{st}}\right)$, and CRP $(\mathrm{mg} / \mathrm{l})$ were assessed. The number of damaged joints was assessed at baseline and 1 year.

Cardiovascular assessment. Anthropomorphic assessments were performed at baseline, every 3 months, and at the end of the study, including height, weight, waist and hip circumferences, 2 consecutive blood pressure (BP) readings in sitting position, and heart rate. Laboratory assessments at each visit included fasting blood glucose, fasting lipid profile [total cholesterol, low-density lipoprotein cholesterol, high-density lipoprotein (HDL) cholesterol, triglycerides], fibrinogen, and uric acid.

$P W V$ and pulse wave analysis (PWA). Arterial stiffness was assessed by PWV and AIx at baseline and at the end of the study ${ }^{11}$. To ensure the consistency and reliability, such assessment for all participants was performed at PWH by a single experienced operator who was blind to the participants clinical characteristics and their treatment group. Brachial-ankle PWV was assessed noninvasively in subjects in the supine position by a dedicated tonometry system (Non-invasive Vascular Profile Device VP-2000, Omron Healthcare Inc.) as described previously ${ }^{12}$. All PWA measurements were made by a single skilled operator. Intraobserver reliability was $0.86^{12}$.

PWA was performed using the SphygmoCor device (SCOR2000 v. 7.01, AtCor Medical Pty. Ltd.), with a tonometer probe at the right radial artery. Because AIx in an individual patient varies by heart rate, it was standardized to a heart rate of 75 bpm (AIx@75). The Vicorder machine (VI, SMT

Personal non-commercial use only. The Journal of Rheumatology Copyright @ 2018 . All rights reserved. 
Medical) was used for AIx assessment since March 2014 because of machine failure (SphygmoCor) after commencement of the study. We were unable to obtain baseline AIx measurement in 26 patients (when the SphygmoCor device was undergoing repair), and 11 patients with baseline assessment done using SphygmoCor were unable to have repeat assessment at Month 12 using the same instrument (after switching to the Vicorder device). Therefore, the AIx results of these 37 patients were excluded from analysis.

Outcome measures. The primary outcome measure was the change in PWV over 1 year of treatment. Secondary outcomes were (1) changes in AIx@75 over 1 year of treatment; (2) the proportion of patients achieving clinical remission $(\mathrm{SDAI} \leq 3.3$ or DAS28 $<2.6$ ) at the end of the study; (3) the proportion of patients with a good response (EULAR definition of DAS28 score $\leq 3.2$ and a fall in score from baseline by > 1.2); and (4) ACR 20, 50, and 70 responses. The outcome for the posthoc analysis was to ascertain the independent predictors for the change in PWV.

Statistical analysis. Descriptive statistics used for demographic and clinical variables included frequencies, percentage, means $\pm \mathrm{SD}$, and median (interquartile range). Comparisons in demographic and clinical characteristics at baseline and changes in AIx@75 and PWV over 1 year between the 2 groups were performed using the chi-squared test, independent samples t test, or the Mann-Whitney U test, depending on distribution of data. Disease activity measures at 12 months were analyzed according to the intention-to-treat (ITT) principle in all individuals with at least 1 additional visit after the baseline. Missing data at the end of the study were accounted for using last observation carried forward. Per-protocol analysis was performed to assess the differences in the progression of vascular stiffness between the 2 groups. A posthoc analysis was done to ascertain the independent predictors associated with the change in PWV using linear regression analysis. Univariate analysis was performed to elucidate whether achieving sustained DAS28 and SDAI remission (defined as achieving DAS28 or SDAI remission at months 6,9 , and 12), other baseline clinical variables (Table 1), and treatment received were associated with the change in PWV. All variables with $\mathrm{p}<0.1$ in the univariate analysis were included in the multivariate analysis. Statistical analyses were performed using SPSS version 23.0 (SPSS Inc.). A minimal level of significance of $\mathrm{p}<0.05$ was used.

\section{RESULTS}

ITT analysis. One hundred twenty patients with early RA were randomized (Figure 1). All except 3 patients were included in the ITT and safety analyses $(n=117)$. All baseline variables were comparable between the groups, except for a higher prevalence of female patients, and lower waist-hip ratio and fasting glucose in Group 1 (Table 1). The 1-year study was completed by 115 patients $(96 \%)$; there were no significant differences between the 2 groups for completion or for followup duration.

Treatments. The proportions of patients receiving MTX monotherapy were lower in Group 1 because most patients received combination csDMARD throughout the study period. The use of prednisolone was significantly lower in Group 1 from 9 months onward (Figure 2A-2E).

Remission and compliance with the protocol. The proportion of patients reaching the respective treatment targets was significantly higher in Group 2, except at 3 months (Figure $2 \mathrm{~F})$. Regarding DAS28 and SDAI remission, the proportion of patients achieving these targets was numerically higher in Group 1 except at Month 12 (Figure 2G and 2H). In Group 1, 37 patients and in Group 2, 20 patients were not given
bDMARD after combination csDMARD therapy failed to achieve targets, because patients were unable to afford the medication.

Changes in disease activity and inflammatory markers. At 12 months, the proportion of patients achieving DAS28 and SDAI remission, various ACR responses, and EULAR good response, as well as the reduction in disease activity and inflammatory markers, were comparable between the 2 groups (Table 2).

Changes in cardiovascular risk factors and arterial stiffness markers. PWV and AIx were similar at both baseline and Month 12 between the 2 groups. Per-protocol analysis revealed that there were no significant between-group differences in the change in PWV and AIx (Table 2). Changes in the $\mathrm{CV}$ risk factors were similar between the 2 groups, except for the changes in systolic BP, diastolic BP, and the Framingham risk score, which were significantly different between the 2 groups (Table 2). This difference is probably due to the more frequent use of antihypertensive drugs in Group 2 (10 patients in each group at baseline; 13 patients in Group 1 vs 16 patients in Group 2 at Month 12, $\mathrm{p}=$ not significant).

Adverse events (AE). Serious (S-) AE were reported in 6/117 patients $(5 \%)$. Three $(5 \%)$ occurred in both Groups 1 and 2 (Supplementary Table 1, available with the online version of this article). All required hospitalization, with none of the SAE being life-threatening. No unexpected SAE or deaths were observed.

Posthoc analysis. Because there were no differences in the change in clinical and vascular variables between the 2 groups (Table 2), a posthoc analysis was performed by combining the results from the 2 groups $(n=110)$, with PWV done to ascertain the independent predictors associated with progression of PWV.

Clinical features in the whole cohort. Out of the 110 patients with RA, 85 (77\%) were female (Table 3). After 12 months, $60(55 \%)$ and $44(40 \%)$ patients achieved DAS28 and SDAI remission, respectively. Although total cholesterol and HDL cholesterol were significantly increased, PWV and AIx remained unchanged over a period of 12 months.

Remission at all 3 timepoints (months 6, 9, and 12) was achieved in $37(34 \%)$ and $18(16.4 \%)$ of patients for DAS28 and SDAI, respectively. The change in PWV was significantly different between the groups who achieved sustained DAS28 remission (SDR group) compared to those who did not (non-SDR group; Table 2). The change in AIx was similar between the SDR and non-SDR groups. The change in PWV and AIx was similar between the groups who achieved sustained SDAI remission compared to those who did not (PWV: $-48 \pm 134 \mathrm{~cm} / \mathrm{s}$ vs $0.4 \pm 193 \mathrm{~cm} / \mathrm{s}$, respectively, $\mathrm{p}=0.313$; AIx: $0.69 \pm 8.15 \%$ vs $-0.43 \pm 7.92 \%$, respectively, $\mathrm{p}=0.649)$.

Baseline clinical characteristics, disease activity, and CV risk factors and arterial stiffness markers between the SDR

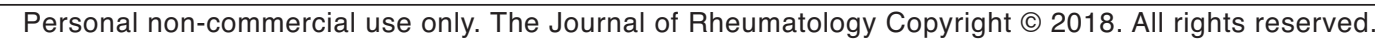




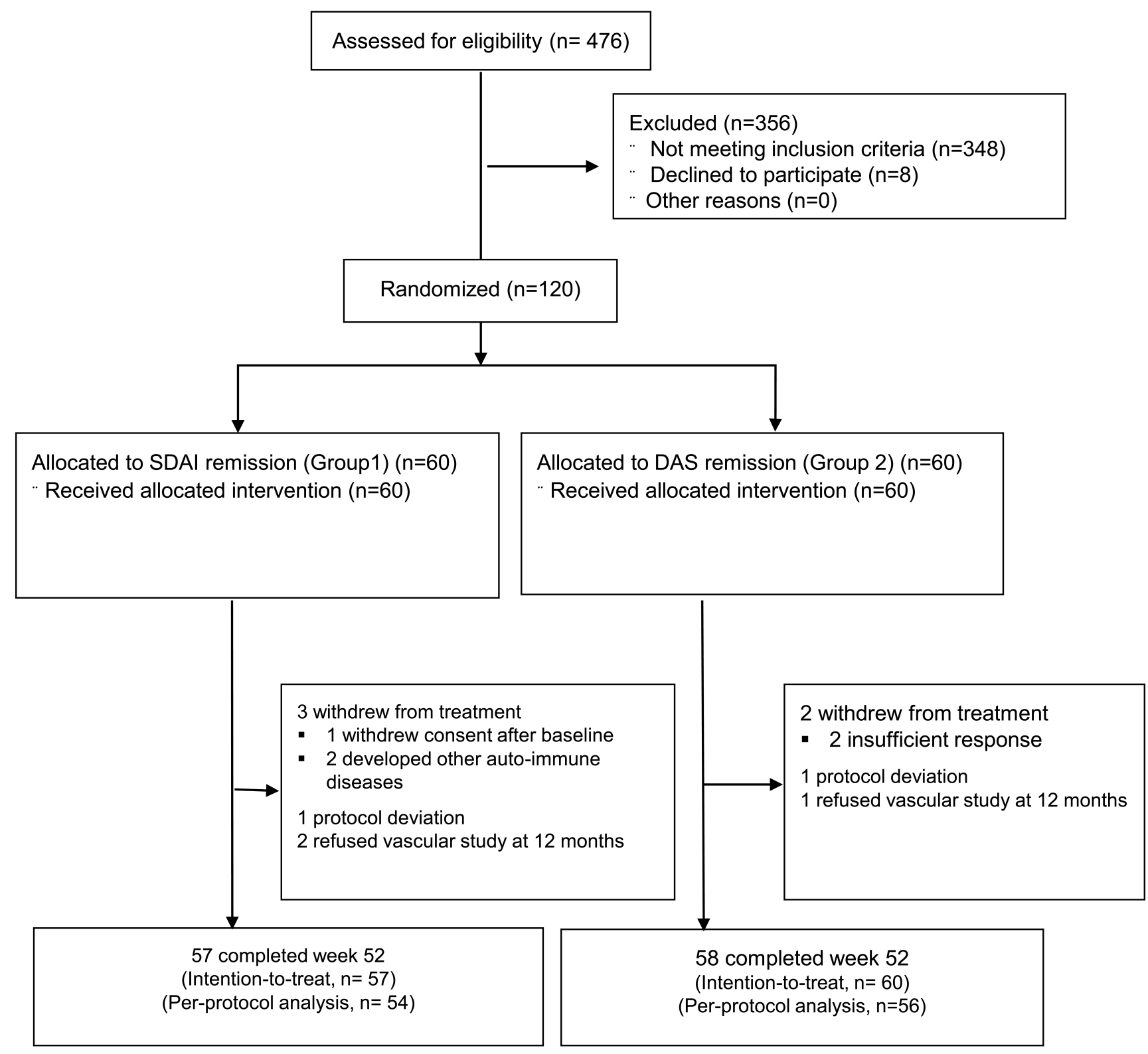

Figure 1. Study flow diagram. SDAI: Simplified Disease Activity Index; DAS: Disease Activity Score.

group and the non-SDR group were similar (Table 1), except that the symptom duration in SDR group was significantly shorter. After 12 months, the SDR group had a significantly greater reduction in disease activity indices and functional improvement (HAQ) compared to the non-SDR group (Table 2), while no significant change in CV risk factors was found between the 2 groups. The change in AIx was similar between the 2 groups.

Predictors associated with the change in PWV. Using multivariate regression analysis, achieving sustained DAS28 remission was an independent predictor for PWV reduction $(\beta-73,95 \%$ CI -150.1 to $-9.1, p=0.027$; Table 4$)$. The other independent predictor for PWV reduction was a shorter symptom duration $(\beta 6.3,95 \%$ CI $0.6-12.0, \mathrm{p}=0.032)$.

\section{DISCUSSION}

To our knowledge, this is the first RCT comparing the clinical and vascular effect of 2 different tight control treatment strategies aiming at SDAI remission and minimal disease activity (DAS28 remission). Although there were no significant differences in the clinical and vascular outcomes between the 2 groups, posthoc analysis showed that patients 
Table 1. Baseline clinical characteristics of patients in the intention-to-treat and posthoc analysis. Values are $\mathrm{n}(\%)$, median (IQR), or mean $\pm \mathrm{SD}$

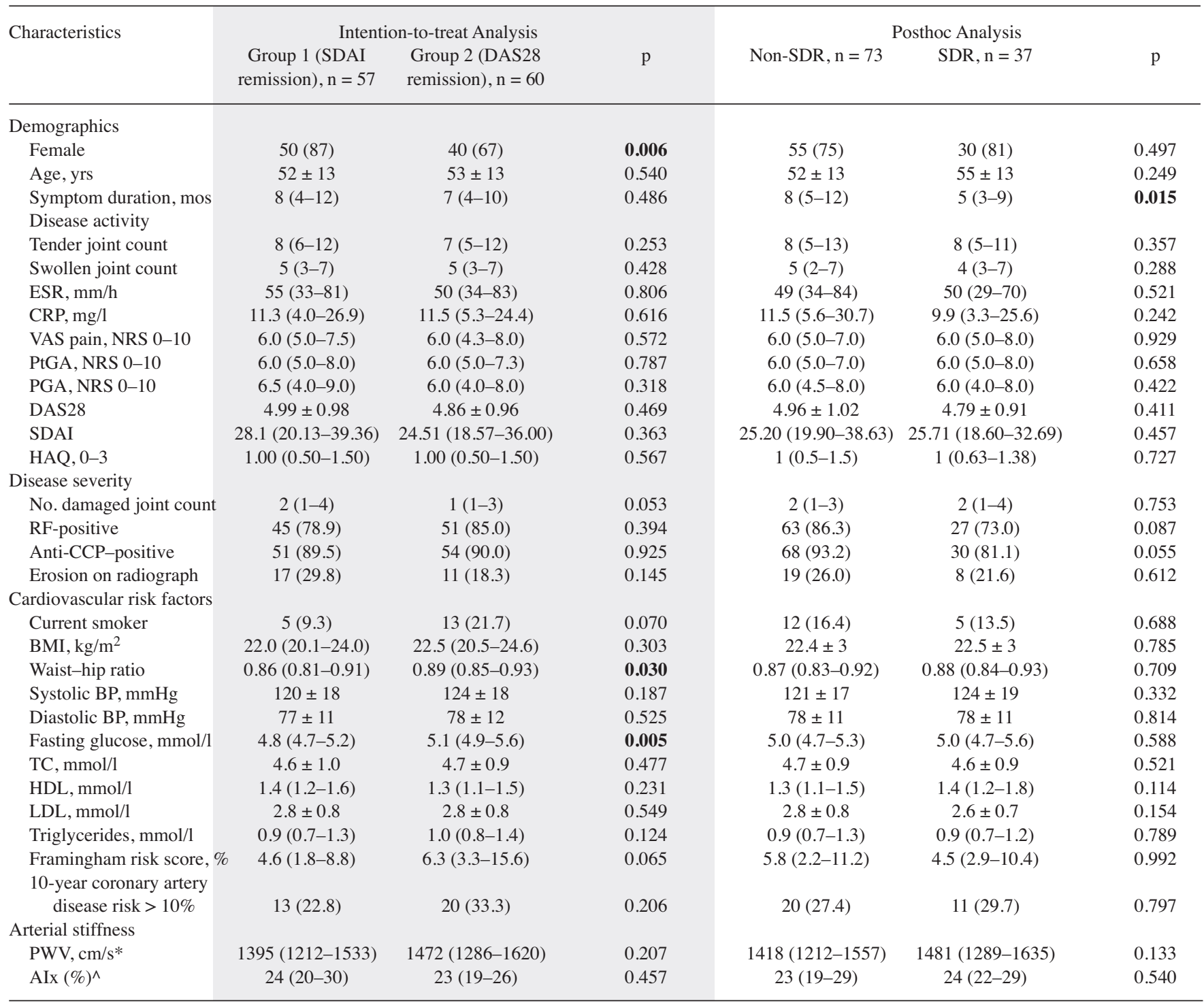

* Per-protocol analysis (Group $1=54$; Group $2=56$ ); posthoc analysis (non-SDR: 73, SDR: 37); ^ Per-protocol analysis (Group 1: 31, Group 2: 38); posthoc analysis (non-SDR: 44, SDR: 25). Data in bold face are statistically significant. IQR: interquartile range; SDAI: Simplified Disease Activity Index; DAS28: 28-joint count Disease Activity Score; SDR: achieved sustained DAS28 remission; ESR: erythrocyte sedimentation rate; CRP: C-reactive protein; VAS: visual analog scale; NRS: numerical rating scale; PtGA: patient's global assessment; PGA: physician's global assessment; HAQ: Health Assessment Questionnaire; RF: rheumatoid factor; anti-CCP: anticyclic citrullinated peptide antibodies; BMI: body mass index; BP: blood pressure; TC: total cholesterol; HDL: high-density lipoprotein; LDL: low-density lipoprotein; PWV: pulse wave velocity; AIx: augmentation index.

who achieved sustained DAS28 remission had a greater reduction in PWV, suggesting that achieving sustained DAS28 remission in early RA may be beneficial not only because of the observed improvement in pain and function, but also because of a reduction in CV risk in terms of preventing arterial stiffness progression. Regarding achieving remission and arterial stiffness, only 1 cross-sectional study demonstrated that patients with active RA had a significantly higher PWV than patients in remission ${ }^{11}$. These results link inflammatory activity to markers of CVD risk in patients with RA and may indirectly support the notion that remission in
RA confers diminished CV morbidity. Indeed, based on the data from a large RA database, a significant trend was observed toward a reduced risk of $\mathrm{CV}$ events with improved disease activity $^{13}$. A population-based cohort of RA patients without CVD also showed a detrimental role of exposure to RA flare and cumulative burden of RA disease severity in CVD risk in $\mathrm{RA}$, while the CVD risk for patients with RA in remission was similar to that for the non-RA subjects ${ }^{7}$. These results add significant new information about the importance of sustained control of RA disease activity, not only for improvement in pain and function, but also for reduced $\mathrm{CV}$ risk.

Personal non-commercial use only. The Journal of Rheumatology Copyright @ 2018 . All rights reserved. 


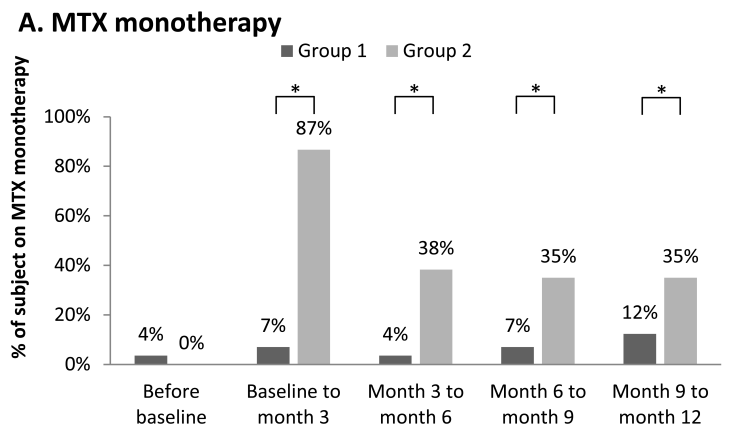

\section{Prednisolone}

\section{aroup 1 -Group 2}

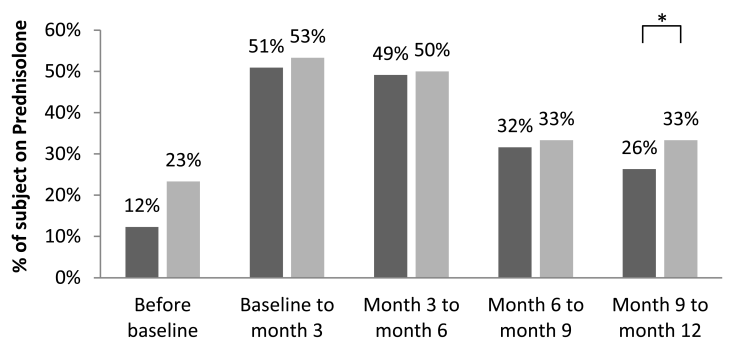

\section{E. bDMARDs}

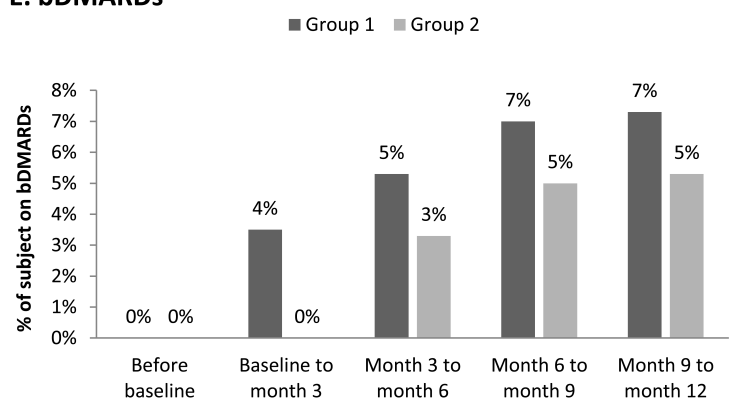

\section{G. Achieved DAS28 remission}

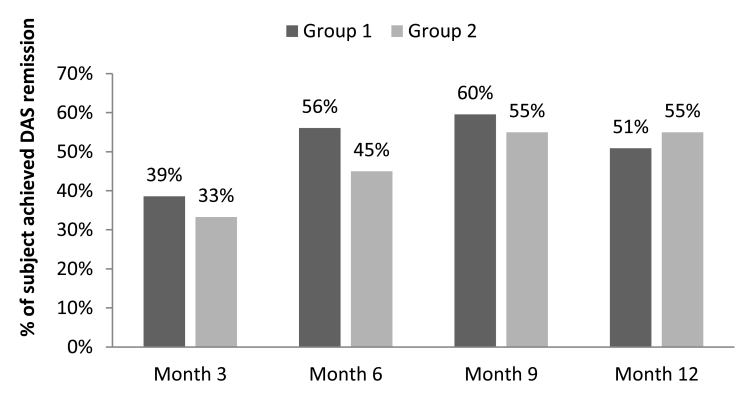

\section{Drug escalation rate}

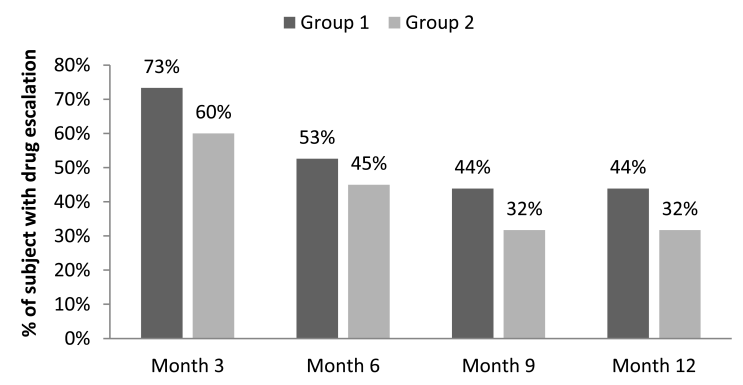

\section{B. csDMARD combination}

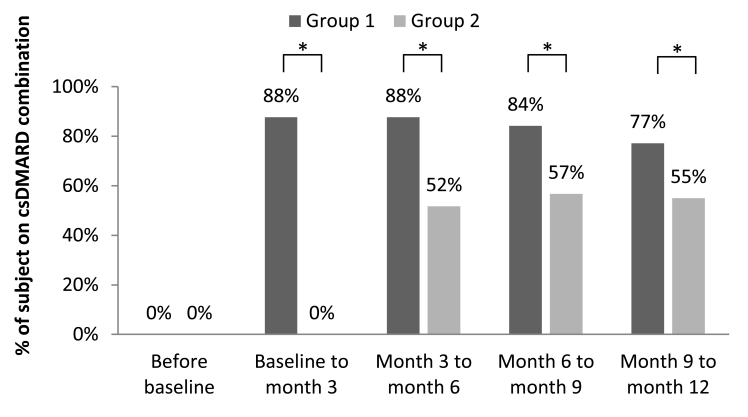

D. NSAIDs

$$
\text { -Group } 1 \text { Group } 2
$$

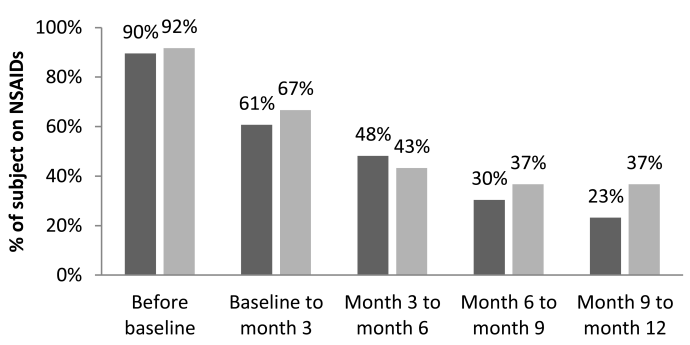

\section{F. Achieved treatment target}

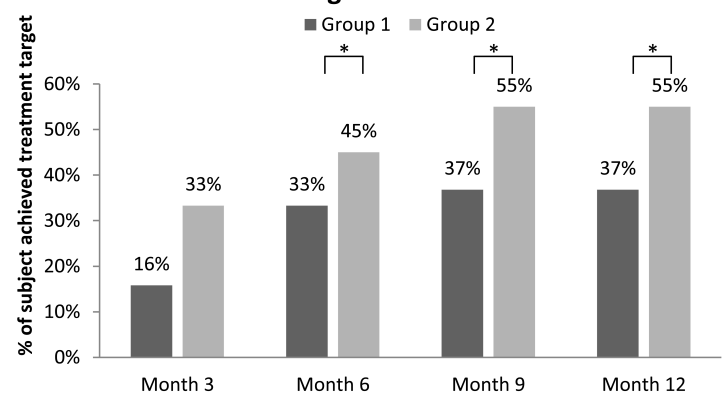

\section{H. Achieved SDAI remission}

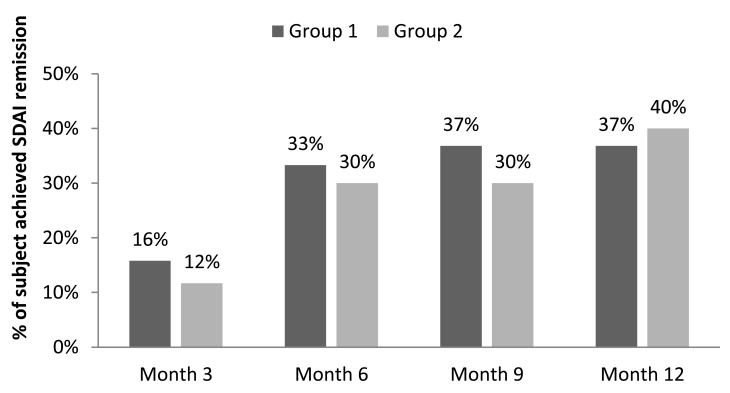

Figure 2. Treatment therapy across study period (A-E) and clinical responses in the 2 groups (F-I). A. MTX monotherapy. B. Combination csDMARD. C. Prednisolone. D. NSAID. E. bDMARD. F. Proportion of subjects achieving treatment target in the 2 randomized groups. $\mathrm{G}$ and $\mathrm{H}$. Proportion of subjects achieving DAS28 and SDAI remission, respectively. I. Proportion of subjects with drug escalation when treatment targets were not met. * (all panels) $p<0.05$ comparing patients who were randomized to treatment strategies aiming at SDAI remission (Group 1) and DAS28 remission (Group 2). MTX: methotrexate; csDMARD: conventional synthetic disease-modifying antirheumatic drugs; NSAID: nonsteroidal antiinflammatory drugs; bDMARD: biologic DMARD; SDAI: Simplified Disease Activity Index; DAS28: 28-joint count Disease Activity Score. 
Table 2. Change in disease activity variables and cardiovascular risk factors over 12 months. Data are median (interquartile range) or mean \pm SD.

\begin{tabular}{|c|c|c|c|c|c|c|}
\hline \multirow[t]{2}{*}{ Variables } & \multicolumn{3}{|c|}{ Intention-to-treat Analysis } & \multicolumn{3}{|c|}{ Posthoc Analysis } \\
\hline & $\begin{array}{l}\text { Group } 1 \text { (SDAI } \\
\text { remission group), } \\
\quad \mathrm{n}=57\end{array}$ & $\begin{array}{l}\text { Group } 2 \text { (DAS } \\
\text { remission group), } \\
\quad n=60\end{array}$ & $\mathrm{p}$ & Non-SDR, $\mathrm{n}=73$ & $\mathrm{SDR}, \mathrm{n}=37$ & $\mathrm{p}$ \\
\hline \multicolumn{7}{|l|}{ Disease activity } \\
\hline Tender joint count & $-6(-10$ to -4$)$ & $-6(-8$ to -3$)$ & 0.501 & $-5(-9$ to -3$)$ & $-7(-10$ to -4$)$ & 0.113 \\
\hline Swollen joint count & $-4(-7$ to -2$)$ & $-3(-6$ to -2$)$ & 0.194 & $-4(-6$ to -2$)$ & $-4(-6$ to -3$)$ & 0.608 \\
\hline $\mathrm{ESR}, \mathrm{mm} / \mathrm{h}$ & $-24(-38$ to -9$)$ & $-22(-32$ to -3$)$ & 0.279 & $-23(-38$ to -3$)$ & $-19(-32$ to -9$)$ & 0.702 \\
\hline CRP, mg/l & $-8.0(-24.6$ to -1.3$)$ & $-8.9(-20.1$ to -1.5$)$ & 0.974 & $-8.0(-19.2$ to -1.5$)$ & $-8.5(-22.5$ to -1.3$)$ & 0.849 \\
\hline VAS pain, NRS 0-10 & $-4.0(-5.5$ to -3.0$)$ & $-4.0(-5.3$ to -2.0$)$ & 0.580 & $-3.8(-5.0$ to -1.5$)$ & $-5.0(-6.5$ to -3.0$)$ & 0.002 \\
\hline PtGA, NRS 0-10 & $-4.0 \pm 2.5$ & $-3.8 \pm 2.4$ & 0.760 & $-3.2 \pm 2.2$ & $-5.3 \pm 2.2$ & $<0.001$ \\
\hline PGA, NRS 0-10 & $-4.5(-7.0$ to -3.0$)$ & $-4.0(-6.3$ to -2.0$)$ & 0.308 & $-4.0(-6.5$ to 2.0$)$ & $-5.0(-7.5$ to -3.5$)$ & 0.026 \\
\hline DAS28 & $-2.47 \pm 1.28$ & $-2.31 \pm 1.28$ & 0.509 & $-2.05 \pm 1.21$ & $-3.18 \pm 0.97$ & $<0.001$ \\
\hline SDAI & $-22.2 \pm 12.62$ & $-20.04 \pm 11.80$ & 0.348 & $-19.47 \pm 12.82$ & $-24.7 \pm 10.11$ & 0.033 \\
\hline HAQ,0-3 & $-0.63(-1.13$ to -0.38$)$ & $-0.50(-1.13$ to 0.00$)$ & 0.247 & $-0.50(-1.13$ to -0.13$)$ & $-0.88(-1.25$ to -0.50$)$ & 0.021 \\
\hline \multicolumn{7}{|c|}{ Cardiovascular risk factors } \\
\hline $\mathrm{BMI}, \mathrm{kg} / \mathrm{m}^{2}$ & $0.20(-0.41$ to 0.79$)$ & $0.27(-0.71$ to 1.35$)$ & 0.647 & $0.18(-0.47$ to 0.95$)$ & $0.30(-0.30$ to 1.08$)$ & 0.324 \\
\hline Waist-hip ratio & $0.01(-0.02$ to 0.02$)$ & $0.01(-0.02$ to 0.04$)$ & 0.391 & $0.01(-0.02$ to 0.03$)$ & $0.01(-0.02$ to 0.05$)$ & 0.694 \\
\hline Systolic BP, mmHg & $1(-7$ to 11$)$ & $-6(-14$ to 0$)$ & 0.002 & $-2(-9$ to 6$)$ & $-6(-14$ to 6$)$ & 0.295 \\
\hline Diastolic BP, mmHg & $-1(-6$ to 6$)$ & $-5(-10$ to -1$)$ & 0.022 & $-4(-8$ to 3$)$ & $-1(-8$ to 3$)$ & 0.512 \\
\hline Fasting glucose, mmol/1 & $0.00(-0.20$ to 0.20$)$ & $0.00(-0.30$ to 0.30$)$ & 0.672 & $0.0(-0.2$ to 0.2$)$ & $-0.1(-0.3$ to 0.2$)$ & 0.276 \\
\hline $\mathrm{TC}, \mathrm{mmol} / \mathrm{l}$ & $0.30(-0.10$ to 0.60$)$ & $0.10(-0.40$ to 0.75$)$ & 0.326 & $0.3(-0.2$ to 0.7$)$ & $0.2(-0.2$ to 0.6$)$ & 0.664 \\
\hline $\mathrm{HDL}, \mathrm{mmol} / \mathrm{l}$ & $0.20(0.00-0.50)$ & $0.20(0.00-0.50)$ & 0.905 & $0.2(0.0-0.5)$ & $0.1(0.0-0.4)$ & 0.269 \\
\hline $\mathrm{LDL}, \mathrm{mmol} / \mathrm{l}$ & $0.00(-0.30$ to 0.30$)$ & $-0.10(-0.50$ to 0.60$)$ & 0.532 & $-0.1(-0.4$ to 0.5$)$ & $0.0(-0.3$ to 0.3$)$ & 0.590 \\
\hline Triglycerides, mmol/1 & $0.10(-0.10$ to 0.40$)$ & $0.00(-0.20$ to 0.30$)$ & 0.502 & $0.0(-0.2$ to 0.3$)$ & $0.0(-0.1$ to 0.2$)$ & 0.626 \\
\hline $\begin{array}{l}\text { Framingham risk score, } \% \\
\text { Arterial stiffness }\end{array}$ & $\% \quad 0.1(-0.8$ to 1.0$)$ & $-0.6(-3.6$ to 0.6$)$ & 0.043 & $-0.12(-1.72$ to 0.53$)$ & $-0.22(-2.3$ to 0.83$)$ & 0.922 \\
\hline $\mathrm{PWV}, \mathrm{cm} / \mathrm{s}^{* \wedge}$ & $16 \pm 160$ & $-30 \pm 204$ & 0.189 & $25 \pm 183$ & $-71 \pm 172$ & 0.009 \\
\hline AIx $\% *^{* \dagger}$ & $0(-5$ to 5$)$ & $-2(-7$ to 5$)$ & 0.708 & $1(-5.5$ to 6$)$ & $-2(-6$ to 4$)$ & 0.255 \\
\hline \multicolumn{7}{|l|}{ Clinical response at $12 \mathrm{mos}$} \\
\hline DAS28 remission & $29(50.9)$ & $33(55.0)$ & 0.655 & $23(31.5)$ & $37(100)$ & $<0.001$ \\
\hline SDAI remission & $21(36.8)$ & $24(40.0)$ & 0.726 & $14(19.2)$ & $30(81.1)$ & $<0.001$ \\
\hline ACR20 & $47(82.5)$ & $49(81.7)$ & 0.911 & $57(78.1)$ & $36(97.3)$ & 0.008 \\
\hline ACR50 & $38(66.7)$ & $40(66.7)$ & 0.999 & $41(56.2)$ & $35(94.6)$ & $<0.001$ \\
\hline ACR70 & $30(52.6)$ & $32(53.3)$ & 0.939 & $28(38.4)$ & $33(89.2)$ & $<0.001$ \\
\hline EULAR good response & $43(75.4)$ & $41(68.3)$ & 0.393 & $44(60.3)$ & $37(100)$ & $<0.001$ \\
\hline
\end{tabular}

* Data from 110 patients who completed Month 12 followup were analyzed. ^ Group 1: 54, Group 2: 56. ${ }^{\dagger}$ Group 1: 31 , Group 2: 38 for the per-protocol analysis; non-SDR: 44, SDR: 25 for the posthoc analysis. Data in bold face are statistically significant. SDAI: Simplified Disease Activity Index; DAS28: 28-joint count Disease Activity Score; SDR: achieved sustained DAS28 remission; ESR: erythrocyte sedimentation rate; CRP: C-reactive protein; VAS: visual analog scale; NRS: numerical rating scale; PtGA: patient's global assessment; PGA: physician's global assessment; HAQ: Health Assessment Questionnaire; BMI: body mass index; BP: blood pressure; TC: total cholesterol; HDL: high-density lipoprotein; LDL: low-density lipoprotein; PWV: pulse wave velocity; AIx: augmentation index. ACR20: American College of Rheumatology 20\% response; EULAR: European League Against Rheumatism.

Managing RA with a treat-to-target (T2T) strategy aiming at remission optimizes clinical, functional, and radiographic outcomes ${ }^{14}$. Regarding the vascular changes, previous studies mainly focused on the effects of bDMARD on the progression of arterial stiffness in $\mathrm{RA}^{15}$. These studies were unable to determine whether the improvement or stabilization of PWV was due to the suppression of inflammation or the blockade of the specific pathways. In contrast, the majority of the patients in our current study was treated only with csDMARD. Data from our study demonstrated that a greater reduction in PWV was observed in those patients achieving sustained DAS28 remission, suggesting that controlling disease activity using various combinations of steroids, csDMARD, and bDMARD may be useful in preventing arterial stiffness progression.
Numerically, a reduction in PWV was observed in those who achieved sustained DAS28 or SDAI remission. The change was statistically insignificant in the sustained SDAI remission group, probably because of the small number of patients achieving this stringent definition of remission $(\mathrm{n}=18)$. The univariate regression coefficient for the change in PWV was numerically smaller (achieving SDAI remission: -48.2 vs achieving DAS remission: -96.2). However, these results would need to be confirmed in larger studies. Physician's global assessment (PGA) was included in the calculation of SDAI but not in DAS28. However, it was not associated with the change in PWV in the univariate analysis (Table 4). This suggested that the inclusion of PGA in determining SDAI remission could not solely explain the differences in the change in PWV between the 2 remission criteria.

Personal non-commercial use only. The Journal of Rheumatology Copyright @ 2018 . All rights reserved. 
Table 3. Clinical characteristics of all patients at baseline and Month 12. Values are $\mathrm{n}(\%)$, median (IQR), or mean \pm SD unless otherwise specified.

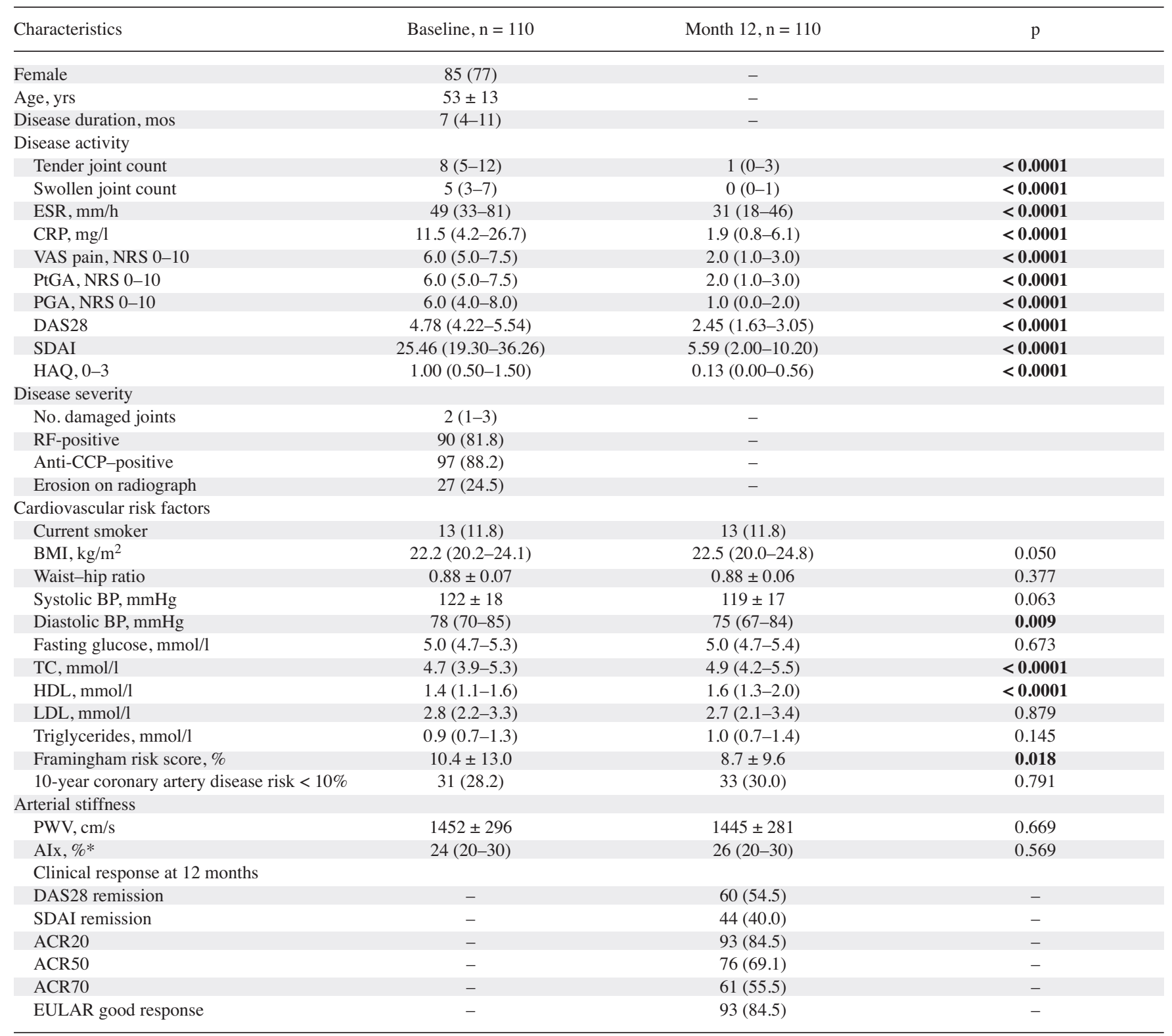

* Baseline: 79, Month 12: 95. Data in bold face are statistically significant. ESR: erythrocyte sedimentation rate; CRP: C-reactive protein; VAS: visual analog scale; NRS: numerical rating scale; DAS28: 28-joint count Disease Activity Score; SDAI: Simplified Disease Activity Index; PtGA: patient's global assessment; PGA: physician's global assessment; HAQ: Health Assessment Questionnaire; RF: rheumatoid factor; anti-CCP: anticyclic citrullinated peptide antibodies; BMI: body mass index; BP: blood pressure; TC: total cholesterol; HDL: high-density lipoprotein; LDL: low-density lipoprotein; PWV: pulse wave velocity; AIx: augmentation index; ACR20: American College of Rheumatology 20\% response; EULAR: European League Against Rheumatism.

A recent study by Arts, et al reported that low disease activity (LDA) is sufficient to achieve a protective effect against CVD in $\mathrm{RA}^{16}$. In view of this recent publication, further analysis was done by dividing patients into 2 groups: sustained LDA group (DAS $\leq 3.2$ at months 6,9, and 12) and moderate to high disease activity group. A trend of reduction in PWV was observed in patients with sustained LDA, although it was not significantly different $(-33.0 \pm 167.4 \mathrm{~cm} / \mathrm{s}$ vs $23.6 \pm 200.8 \mathrm{~cm} / \mathrm{s}$, respectively, $\mathrm{p}=0.113)$. The results were similar when the LDA was defined by $\operatorname{SDAI}(\leq 11$; $-33.1 \pm 164.2 \mathrm{~cm} / \mathrm{s}$ vs $24.3 \pm 204.1 \mathrm{~cm} / \mathrm{s}$, respectively, $\mathrm{p}=0.106$ ). Data from Arts, et $\mathrm{l}^{16}$, as well as from our study, showed that patients who achieve and maintain remission or LDA during followup may be less likely to develop bouts of uncontrolled, sustained, high systemic inflammation, a contributing factor to atherosclerosis and CVD. RA patients with very active disease at diagnosis, and poor treatment response with more frequent flares as a result, may form a 
Table 4. Regression analysis for the change in PWV after 1 year.

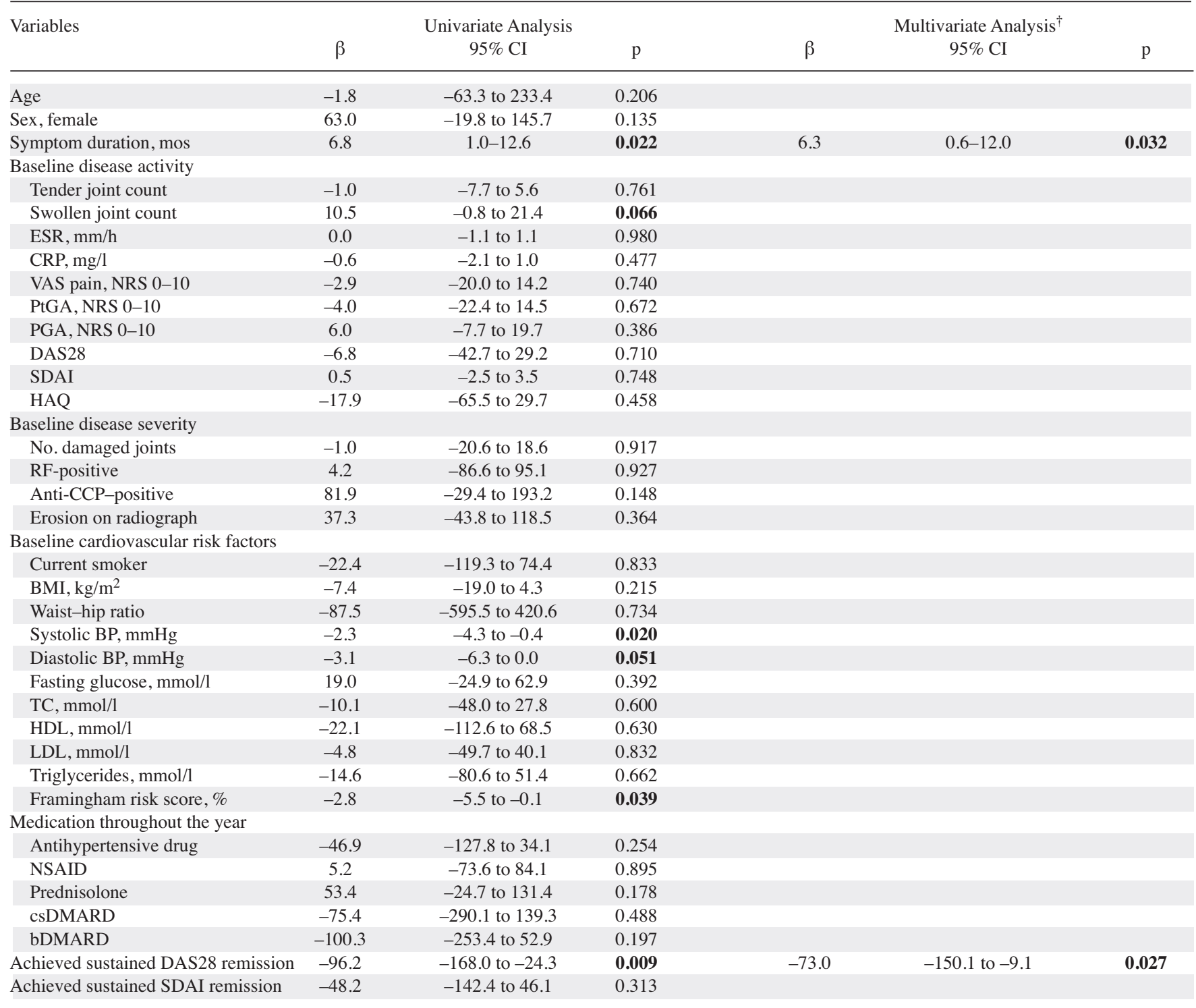

$\dagger$ Adjusted for age, sex, symptom duration, swollen joint count, systolic and diastolic BP, and Framingham risk score. Data in bold face are statistically significant. PWV: pulse wave velocity; ESR: erythrocyte sedimentation rate; CRP: C-reactive protein; VAS: visual analog scale; NRS: numerical rating scale; PtGA: patient's global assessment; PGA: physician's global assessment; DAS28: 28-joint count Disease Activity Score; SDAI: Simplified Disease Activity Index; HAQ: Health Assessment Questionnaire; RF: rheumatoid factor; anti-CCP: anticyclic citrullinated peptide antibodies; BMI: body mass index; BP: blood pressure; TC: total cholesterol; HDL: high-density lipoprotein; LDL: low-density lipoprotein; NSAID: nonsteroidal antiinflammatory drugs; csDMARD: conventional synthetic disease-modifying antirheumatic drugs; bDMARD: biologic DMARD.

subgroup within the RA population that is particularly at risk for developing CVD, significantly contributing to the excess CVD risk in this population ${ }^{16}$.

Because of the limited availability of bDMARD, the main differences in the treatment between the 2 groups included a significantly higher proportion of patients who received combination csDMARD in the SDAI remission group, while more patients received MTX monotherapy in the DAS28 remission group throughout the study period. Despite that, DAS28 remission was achieved in around $50 \%$ of patients in both groups, similar to other DAS-driven trials in early
RA $^{17,18,19,20}$. Our results are similar to those from the CARERA study and the tREACH trial: MTX monotherapy is not inferior to combination csDMARD therapy when used in combination with glucocorticoids following a tight T2T approach $^{21,22}$. To fully address whether obtaining more stringent remission results in a larger reduction in arterial stiffness, future studies may consider randomizing patients in DAS28 remission but not SDAI remission to additional placebo or intensified treatment, especially if funding is available to provide bDMARD if patients cannot achieve SDAI remission.

\section{Personal non-commercial use only. The Journal of Rheumatology Copyright @ 2018. All rights reserved.}


In our current study, no differences in the change in AIx in patients were found. One possibility could be related to the small sample size; the data of 37 patients were not analyzable. However, these results were similar to those of most studies on antitumor necrosis factor (TNF), which demonstrated therapeutic effect on PWV (improved or stable in anti-TNF treatment group while worsened in control group); such an effect was not seen on AIx ${ }^{15}$. These data suggest that PWV may be more responsive to change after controlling inflammation (either by anti-TNF or by achieving sustained remission) in patients with chronic inflammatory arthritis.

The strength of our study was the inclusion of patients with short disease duration in whom changes in the vessel wall may reflect current (but reversible) inflammation rather than more permanent structural vessel changes. There were several limitations of our trial. First, in Hong Kong, bDMARD are not reimbursed by the government. As a result, most patients treated with bDMARD had to cover the medication costs themselves. This may explain why few patients were able to afford treatment with bDMARD after failing combination csDMARD. A previous study reported that with early initiation of tocilizumab with or without MTX, $>80 \%$ of patients with RA achieved sustained remission, compared with $44 \%$ in the MTX $\operatorname{arm}^{23}$. Whether early initiation of bDMARD would improve $\mathrm{CV}$ outcome compared to combination csDMARD needs to be addressed in future studies. Second, we used surrogate endpoints instead of actual CV events. There is only 1 study suggesting that PWV is a good surrogate of future CVD events, specifically in patients with $\mathrm{RA}^{24}$. Last, a more frequent PWV examination might be considered to record early changes in PWV before Month 12 .

With limited access to bDMARD, treatment targets aiming at DAS28 and SDAI remission had a similar effect in preventing the progression of arterial stiffness at 1 year in patients with early RA. However, achieving sustained DAS28 remission improved arterial stiffness in terms of PWV reduction. Adopting a T2T strategy that aims to achieve sustained DAS28 remission may confer benefits on CVD outcomes.

\section{ACKNOWLEDGMENT}

We thank our patients for their time in participating in this project. We also thank Dr. Woon-Leung Ng, Dr. Man-Chi Leung, Dr. Chi-Keung Sung, and Pik Fan Lee for patient referral.

\section{ONLINE SUPPLEMENT}

Supplementary material accompanies the online version of this article.

\section{REFERENCES}

1. Dadoun S, Zeboulon-Ktorza N, Combescure C, Elhai M, Rozenberg $\mathrm{S}$, Gossec L, et al. Mortality in rheumatoid arthritis over the last fifty years: systematic review and meta-analysis. Joint Bone Spine 2013;80:29-33

2. Arts EE, Fransen J, den Broeder AA, Popa CD, van Riel PL. The effect of disease duration and disease activity on the risk of cardiovascular disease in rheumatoid arthritis patients. Ann Rheum Dis 2015;74:998-1003.
3. Innala L, Moller B, Ljung L, Magnusson S, Smedby T, Sodergren A, et al. Cardiovascular events in early RA are a result of inflammatory burden and traditional risk factors: a five year prospective study. Arthritis Res Ther 2011;13:R131.

4. Schipper LG, van Hulst LT, Grol R. Meta-analysis of tight control strategies in rheumatoid arthritis: protocolized treatment has additional value with respect to the clinical outcome. Rheumatology 2010;49:2154-64.

5. Wells GA, Boers M, Shea B, Brooks PM, Simon LS, Strand CV, et al. Minimal disease activity for rheumatoid arthritis: a preliminary definition. J Rheumatol 2005;32:2016-24.

6. Felson DT, Smolen JS, Wells G, Zhang B, van Tuyl LH, Funovits J, et al; American College of Rheumatology; European League Against Rheumatism. American College of Rheumatology/ European League Against Rheumatism provisional definition of remission in rheumatoid arthritis for clinical trials. Arthritis Rheum 2011;63:573-86.

7. Myasoedova E, Chandran A, Ilhan B, Major BT, Michet CJ, Matteson EL, et al. The role of rheumatoid arthritis (RA) flare and cumulative burden of RA severity in the risk of cardiovascular disease. Ann Rheum Dis 2016;75:560-5.

8. Mäki-Petäjä KM, Hall FC, Booth AD, Wallace SM, Yasmin, Bearcroft PW, et al. Rheumatoid arthritis is associated with increased aortic pulse-wave velocity, which is reduced by anti-tumor necrosis factor-alpha therapy. Circulation 2006;114:1185-92.

9. Tam LS, Shang Q, Li EK, Wang S, Li RJ, Lee KL, et al. Infliximab is associated with improvement in arterial stiffness in patients with early rheumatoid arthritis - a randomized trial. J Rheumatol 2012;39:2267-75.

10. Aletaha D, Neogi T, Silman AJ, Funovits J, Felson DT, Bingham $\mathrm{CO}$ 3rd, et al. 2010 rheumatoid arthritis classification criteria: an American College of Rheumatology/European League Against Rheumatism collaborative initiative. Arthritis Rheum 2010; 62:2569-81.

11. Provan SA, Semb AG, Hisdal J, Stranden E, Agewall S, Dagfinrud $\mathrm{H}$, et al. Remission is the goal for cardiovascular risk management in patients with rheumatoid arthritis: a cross-sectional comparative study. Ann Rheum Dis 2011;70:812-7.

12. Tam LS, Li EK, Shang Q, Tomlinson B, Lee VW, Lee KK, et al. Effects of rosuvastatin on subclinical atherosclerosis and arterial stiffness in rheumatoid arthritis: a randomized controlled pilot trial. Scand J Rheumatol 2011;40:411-21.

13. Solomon DH, Reed GW, Kremer JM, Curtis JR, Farkouh ME, Harrold LR, et al. Disease activity in rheumatoid arthritis and the risk of cardiovascular events. Arthritis Rheum 2015;67:1449-55.

14. Smolen JS, Breedveld FC, Burmester GR, Bykerk V, Dougados M, Emery P, et al. Treating rheumatoid arthritis to target: 2014 update of the recommendations of an international task force. Ann Rheum Dis 2016;75:3-15.

15. Shen J, Shang Q, Tam LS. Targeting inflammation in the prevention of cardiovascular disease in patients with inflammatory arthritis. Transl Res 2016;167:138-51.

16. Arts EE, Fransen J, Den Broeder AA, van Riel P, Popa CD. Low disease activity (DAS28 $\leq 3.2$ ) reduces the risk of first cardiovascular event in rheumatoid arthritis: a time-dependent Cox regression analysis in a large cohort study. Ann Rheum Dis 2017;76:1693-9.

17. Schipper LG, Vermeer M, Kuper HH, Hoekstra MO, Haagsma CJ, Den Broeder AA, et al. A tight control treatment strategy aiming for remission in early rheumatoid arthritis is more effective than usual care treatment in daily clinical practice: a study of two cohorts in the Dutch Rheumatoid Arthritis Monitoring registry. Ann Rheum Dis 2012;71:845-50.

18. Goekoop-Ruiterman YP, de Vries-Bouwstra JK, Kerstens PJ, Nielen

Personal non-commercial use only. The Journal of Rheumatology Copyright $\odot$ (2018. All rights reserved 
MM, Vos K, van Schaardenburg D, et al. DAS-driven therapy versus routine care in patients with recent-onset active rheumatoid arthritis. Ann Rheum Dis 2010;69:65-9.

19. Soubrier M, Lukas C, Sibilia J, Fautrel B, Roux F, Gossec L, et al. Disease activity score-driven therapy versus routine care in patients with recent-onset active rheumatoid arthritis: data from the GUEPARD trial and ESPOIR cohort. Ann Rheum Dis 2011; 70:611-5.

20. Shen J, Shang Q, Wong CK, Li EK, Wang S, Li RJ, et al. IL-33 and soluble ST2 levels as novel predictors for remission and progression of carotid plaque in early rheumatoid arthritis: a prospective study. Semin Arthritis Rheum 2015;45:18-27.

21. Verschueren P, De Cock D, Corluy L, Joos R, Langenaken C, Taelman V, et al. Methotrexate in combination with other DMARDs is not superior to methotrexate alone for remission induction with moderate-to-high-dose glucocorticoid bridging in early rheumatoid arthritis after 16 weeks of treatment: the CareRA trial. Ann Rheum Dis 2015;74:27-34.

22. de Jong PH, Hazes JM, Han HK, Huisman M, van Zeben D, van der Lubbe PA, et al. Randomised comparison of initial triple DMARD therapy with methotrexate monotherapy in combination with low-dose glucocorticoid bridging therapy; 1-year data of the tREACH trial. Ann Rheum Dis 2014;73:1331-9.

23. Bijlsma JWJ, Welsing PMJ, Woodworth TG, Middelink LM, Pethö-Schramm A, Bernasconi C, et al. Early rheumatoid arthritis treated with tocilizumab, methotrexate, or their combination (U-Act-Early): a multicentre, randomised, double-blind, double-dummy, strategy trial. Lancet 2016;388:343-55.

24. Ikdahl E, Rollefstad S, Wibetoe G, Olsen IC, Berg IJ, Hisdal J, et al. Predictive value of arterial stiffness and subclinical carotid atherosclerosis for cardiovascular disease in patients with rheumatoid arthritis. J Rheumatol 2016;43:1622-30. 